\title{
Increased Plasma and Renal Clearance of an Exchangeable Pool of Apolipoprotein A-I in Subjects with Low Levels of High Density Lipoprotein Cholesterol
}

\author{
Barry S. Horowitz, * Ira J. Goldberg, * Jacques Merab, * Theresa M. Vanni, ^ Rajasekhar Ramakrishnan,” \\ and Henry N. Ginsberg* \\ *Departments of Medicine and ${ }^{\ddagger}$ Pediatrics, Columbia University College of Physicians and Surgeons \\ and the Columbia Presbyterian Medical Center, New York 10032
}

\begin{abstract}
Plasma levels of HDL apo A-I are reduced in individuals with low HDL cholesterol (HDL-C) concentrations as a result of increased fractional catabolic rates (FCRs). To determine the basis for the high apo A-I FCRs, seven subjects with low HDL$C$ levels (31.0 $\pm 4.3 \mathrm{mg} / \mathrm{dl})$ were compared with three subjects with high HDL-C levels $(72.0 \pm 4.5 \mathrm{mg} / \mathrm{dl})$. Each subject received autologous HDL that was labeled directly by the iodinemonochloride method (whole-labeled) and autologous HDL that was labeled by exchange with homologous radiolabeled apo A-I (exchange-labeled). Blood was obtained for 2 wk, specific activities determined, and FCRs $\left(d^{-1} \pm S D\right)$ estimated. In every subject, whether in the low or high HDL-C group, the exchange-labeled FCR was greater than the whole-labeled FCR. The exchange-labeled FCR was higher in the low HDL-C group (0.339 \pm 0.043$)$ versus the high HDL-C group (0.234 $\pm 0.047 ; P<0.009)$. The whole-labeled FCR was also greater in the low HDL-C group (0.239 \pm 0.023$)$ versus the high HDL$C$ group $(0.161 \pm 0.064 ; P<0.02)$. In addition, in both low and high HDL groups ultracentrifugation resulted in more radioactivity in $d>1.210$ (as percentage of total plasma counts per minute) with the exchange-labeled tracer than with the wholelabeled tracer $(12.55 \pm 4.95 \%$ vs. $1.02 \pm 0.38 \% ; P<0.003)$. With both HDL tracers, more radioactivity was found in $d$ $>1.210$ in the low versus the high HDL-C groups. When apo A-I catabolism was studied by perfusing isolated rabbit kidneys with whole-labeled HDL, there was twice as much accumulation (cpm/g cortex) of HDL apo A-I isolated from subjects with low HDL-C than from subjects with high HDL-C (P $<0.0025)$. Finally, HDL that had been isolated from subjects with high levels of HDL-C was triglyceride enriched and exposed to partially purified lipases before perfusion through kidneys. Threefold more apo A-I from modified HDL accumulated in the cortex compared with the unmodified preparation ( $P$ $<0.007)$. The results of these in vivo and ex vivo studies indicate that individuals with low HDL-C levels have more loosely bound, easily exchanged apo A-I and that this exchangeable apo A-I is more readily cleared by the kidney. (J. Clin. Invest.
\end{abstract}

Address correspondence to Dr. Henry N. Ginsberg, Department of Medicine, P \& S 9-510, College of Physicians and Surgeons, New York, NY 10032.

Received for publication 30 March 1992 and in revised form 3 September 1992.

J. Clin. Invest.

(C) The American Society for Clinical Investigation, Inc.

$0021-9738 / 93 / 04 / 1743 / 10 \$ 2.00$

Volume 91, April 1993, 1743-1752
1993. 91:1743-1752.) Key words: lipoproteins • high density lipoproteins • apolipoprotein A-I • cholesterol • metabolism

\section{Introduction}

Plasma concentrations of HDL cholesterol are significantly and inversely related to risk for developing coronary heart disease (1-3). Individuals with low plasma levels of HDL cholesterol also have reduced concentrations of apo A-I, and low plasma apo A-I levels are also associated with increased risk for coronary heart disease (4). Reduced plasma levels of apo A-I could result from decreased secretion into plasma or increased fractional removal from plasma of this apolipoprotein. Data from several laboratories have demonstrated that increased HDL apo A-I fractional catabolic rates (FCRs) ${ }^{1}$ and not reduced rates of apo A-I secretion into plasma are associated with low apo A-I concentrations (5). Additionally, recent studies indicate that high apo A-I FCRs are associated with low HDL levels in subjects with either hypertriglyceridemia or normal plasma triglyceride concentrations $(6,7)$.

Neither the mechanisms underlying accelerated fractional catabolism nor the sites of increased removal of apo A-I from plasma have been clearly delineated. Of interest in this regard are the studies by Glass et al. $(8,9)$, which demonstrated that the kidney is a major site of HDL apo A-I catabolism and that this organ can accumulate HDL apo A-I without core lipid components. These results suggested uptake and catabolism of "free" apo A-I by that organ. Apo A-I can exchange among lipoprotein particles in vivo (10), indicating that it has the ability to associate with and dissociate from HDL (as well as other lipoproteins). Indeed, free apo A-I has been identified in plasma by several investigators (11-14) and increased free apo A-I is present in plasma of patients with renal failure (15). Apo A-I has been shown to be filtered by the glomerulus (16) and has been measured in urine from normal individuals (17) and from nephrotic patients (18). The quantitative importance of the kidney in apo A-I catabolism was suggested by the recent studies of Goldberg et al. (19), which demonstrated that increased apo A-I FCRs in hypertriglyceridemic monkeys were associated with increased renal catabolism of this apolipoprotein.

On the basis of these data, we hypothesized that increased fractional catabolism of apo A-I in subjects with low plasma HDL cholesterol levels results from increased dissociation of

1. Abbreviations used in this paper: AHA, American Heart Association; CETP, cholesteryl ester transfer protein; FCR, fractional catabolic rate; FPLC, fast performance liquid chromatography; HTGL, hepatic triglyceride lipase; LPL, lipoprotein lipase. 
apo A-I from HDL particles and subsequent clearance of free apo A-I by the kidney. To identify and characterize the metabolism of the loosely bound (or more readily dissociable) pool of apo A-I, we performed turnover studies using a dual-tracer approach. HDL was either radioiodinated directly (whole-labeled) or by exchange with pure apo A-I (exchange-labeled), and these tracers were used to determine apo A-I FCRs in individuals with high and low concentrations of HDL cholesterol. In addition, isolated perfused rabbit kidneys were used to compare renal uptake of HDL isolated from subjects with low and high HDL cholesterol concentrations. Finally, renal uptake of HDL modified by triglyceride enrichment followed by exposure to lipoprotein lipase (LPL) and hepatic triglyceride lipase (HTGL) was also determined. Our results indicate that subjects with low plasma levels of HDL cholesterol have increased apo A-I FCRs because they have more loosely bound, more easily exchanged apo A-I that is more readily cleared by the kidney.

\section{Methods}

Subjects. Men identified as having either low $(<25$ th percentile) or high ( $>90$ th percentile) plasma HDL cholesterol concentrations before any dietary intervention were recruited from a patient population referred to our lipid clinic for evaluation and from staff and students at the Health Sciences Campus of Columbia University. The subjects with low HDL cholesterol levels had been eating an American Heart Association (AHA) Step 1 diet for periods ranging from several weeks to several years. Some of these men had previously been treated with lipid-altering agents but had not received any lipid-lowering agent for $\geq 6$ wk before study. Each subject with a high plasma HDL cholesterol level was instructed in the AHA diet by one of our research dietitians 2-3 wk before study. None of these men had ever received a lipid-altering drug. None of the study subjects in either group had any other disease nor were they taking any medication known to affect lipid metabolism. This study was approved by the Institutional Review Board at our medical center and each subject consented to participate in the study.

Turnover protocol. Blood was obtained by venipuncture after a 12 14-h fast for isolation of lipoproteins. Plasma HDL of density 1.0631.210 was isolated by sequential ultracentrifugation using sterile techniques (7). HDL was whole-labeled with ${ }^{125}$ I, using a modification of the method described by McFarlane (7, 20). Homologous apo A-I was radiolabeled similarly with ${ }^{131} \mathrm{I}$. Exchange-labeled HDL was prepared by incubating the ${ }^{131} \mathrm{I}$-apo A-I with a separate aliquot of the subject's $\mathrm{HDL}$ at $37^{\circ} \mathrm{C}$ for $30 \mathrm{~min}$, followed by reisolation of the HDL by ultracentrifugation. Approximately $20 \mu \mathrm{g}$ of radiolabeled apo A-I was incubated with $500 \mu \mathrm{g}$ of $\mathrm{HDL}$; these quantities were chosen so that the labeled apo A-I would exchange with unlabeled apo A-I without increasing the actual apo A-I content of the lipoprotein (21). The two radiolabeled HDL tracers were injected within $24 \mathrm{~h}$ of preparation.

Subjects were admitted to the General Clinical Research Center (GCRC) $1 \mathrm{~d}$ before injection with the radiolabeled lipoproteins. After an overnight fast, a sample of blood was obtained and $25 \mu \mathrm{Ci}$ of ${ }^{131} \mathrm{I}$-apo A-I-HDL and $75 \mu \mathrm{Ci}$ of ${ }^{125} \mathrm{I}-\mathrm{HDL}$ were injected intravenously. Samples were then obtained at $0.5,1,2,6,12,24$, and $36 \mathrm{~h}$. Daily blood samples were obtained during the next $2 \mathrm{wk}$. Patients stayed in the Clinical Research Center for at least the first $36 \mathrm{~h}$ after injection of tracers. Afterwards, they returned daily for additional fasting blood samples. Subjects consumed the AHA Step 1 diet during their stay in the GCRC and were instructed to continue that diet while completing the study as outpatients. Dietary compliance was monitored closely throughout the study by frequent meetings between the research dietitian and the participants. All subjects received a saturated solution of potassium iodide twice daily, starting the day before tracer injections and continuing throughout the study period to prevent thyroidal accumulation of radioiodine.

At the end of the study, plasma obtained from each blood sample was subjected to sequential ultracentrifugation in a $50.3 \mathrm{Ti}$ rotor to isolate HDL (22). First, VLDL, intermediate density lipoprotein, and LDL were removed together by ultracentrifugation at $39,000 \mathrm{rpm}$ for $24 \mathrm{~h}$ at $d=1.063$. This was followed by a second centrifugation at the same density. $\operatorname{HDL}(d=1.063-1.210)$ was then isolated by ultracentrifugation at $39,000 \mathrm{rpm}$ for $48 \mathrm{~h}$ at $d=1.210$. The isolated HDL was further purified by recentrifugation at the same density for $24 \mathrm{~h} .{ }^{131} \mathrm{I}-$ apo A-I-HDL radioactivity in aliquots of HDL was determined by gamma counting, HDL protein concentration was assayed by the method of Lowry et al. (23), and the exchange-labeled specific radioactivity of HDL apo A-I in each sample was calculated. An aliquot of each HDL sample was also delipidated, redissolved in $6 \mathrm{M}$ urea, and apo A-I isolated from other apolipoproteins by fast performance liquid chromatography (FPLC) $(7,24)$. FPLC purification of apo A-I was necessary for specific radioactivity determinations from whole-labeled HDL because several apolipoproteins were labeled by the IC1 method. The pooled fractions of pure apo A-I were counted, protein concentrations were determined, and whole-label specific radioactivity of HDL apo A-I was calculated. The specific radioactivity data were then used to estimate the FCRs of exchange- and whole-labeled HDL apo A-I, using a two-pool model for each tracer (7), with the proviso that the two models would have the same kinetics for noncirculating HDL (in the extravascular pool). Radioactivity in the $d>1.210$ fraction as a percent of total plasma radioactivity was examined to assess dissociation of apo A-I from HDL that had been isolated from subjects with low versus high HDL cholesterol concentrations. In addition, we measured apo A-I mass in the $d>1.210$ fraction as a percent of HDL apo A-I mass.

Two variations of this protocol were carried out. First, the tracers were reversed in two subjects with low HDL cholesterol levels, so that ${ }^{125}$ I was used for the exchange-labeled tracer and ${ }^{131}$ I was used for the whole-labeled tracer. This was done to determine if there was a "tracer effect" (25) that might account for any differences observed in FCRs. Next, a subject with a low HDL cholesterol level was injected with one exchange-labeled tracer prepared with homologous apo A-I as described above. This individual, however, also received an injection of a second exchange-labeled tracer that had been prepared using the subject's own purified apo A-I. A 20-fold greater mass of autologous apo A-I was labeled compared with homologous apo A-I. This variation of the protocol was undertaken to see if using autologous versus homologous apo A-I resulted in differences in FCRs. As the two tracers had widely differing specific activities, this protocol also allowed us to determine if radiation damage of a high specific activity tracer could explain the differences observed between the FCRs of the two tracers.

In vitro studies. To further assess any possible effects of ${ }^{125}$ I versus ${ }^{131} I$ on the outcome of the in vivo studies, a long-term in vitro incubation protocol was designed. HDL was both whole- and exchange-labeled with both ${ }^{125} I$ and ${ }^{131} I$, thus creating four separate tracers. Two separate incubations were performed with plasma from subject 9 , using ${ }^{131}$ I whole-labeled tracer and ${ }^{125}$ I exchange-labeled tracer in one incubation and ${ }^{125} I$ whole-labeled tracer and ${ }^{131} I$ exchange-labeled tracer in the other. The incubations were carried out at $37^{\circ} \mathrm{C}$ for $2 \mathrm{wk}$. Every $2 \mathrm{~d}$, an aliquot was taken from each incubation and subjected to ultracentrifugation at $d: 1.210$ for $24 \mathrm{~h}$. Radioactivity in the $d<1.210$ and $d>$ 1.210 fractions was examined to assess any radiation damage to particles that might have occurred over the 2-wk period.

Kidney perfusion studies. Renal uptake of apo A-I was studied using kidneys isolated from New Zealand white rabbits and perfused according to the method of Maack (26). The kidney perfusate contained $7.5 \%$ albumin in Krebs-Henseleit buffer and $6 \mathrm{mM}$ glucose, 4 $\mathrm{mM}$ creatinine, $0.05 \mathrm{mM}$ methionine, $2 \mathrm{mM}$ alanine, $2 \mathrm{mM}$ glycine, 2 mM serine, $1 \mathrm{mM}$ arginine, $2 \mathrm{mM}$ proline, $1 \mathrm{mM}$ isoleucine, and $3 \mathrm{mM}$ aspartic acid. There were no erythrocytes, and the partial pressure of $\mathrm{O}_{2}$ was maintained by continuous aeration of the buffer with a $95 \%$ oxygen-5\% carbon dioxide mixture. The hydrostatic pressure of the perfus- 
ate as it entered the kidney was continuously monitored using a polygraph (model 7, Grass Instrument Co., Quincy, MA) and the flow rate of the perfusate was adjusted to maintain the pressure at $90 \mathrm{mmHg}$. By maintaining a perfusion pressure that approximates the in vivo situation, we assumed that the hydrostatic pressure within the glomerulus was also similar to that present in vivo.

Whole-labeled ${ }^{125}$ I-HDL $(d=1.063-1.210)$ from subjects with low or high HDL cholesterol levels was first incubated with excess VLDL for $24 \mathrm{~h}$ at $37^{\circ} \mathrm{C}$. This allowed exchange of labeled HDL apo Cs and apo $\mathrm{E}$ for unlabeled apolipoproteins in VLDL. The reisolated ${ }^{125} \mathrm{I}$ HDL, enriched in radiolabeled apo A-I ( $>80 \%$ of the total radioactivity as determined by SDS-PAGE), was injected into the perfusate and aliquots of venous effluent were collected at 5 and $30 \mathrm{~min}$. After $30 \mathrm{~min}$ the kidney was washed with $5 \mathrm{vol}$ of fresh perfusate to rinse out nonspecifically associated label. The kidney was then weighed and sections of renal cortex were cut out for gamma counting. Uptake of apo A-I by the kidney was quantified by determining the radioactivity in the cortex, expressed per $\mathrm{g}$ tissue. This value was normalized to initial radioactivity in the perfusate. Radiolabeled retinol binding protein (RBP), which is filtered and catabolized by the kidney, was used as a control for glomerular function in each experiment.

Modification of $H D L$. The effects of triglyceride enrichment and lipase treatment on uptake of HDL apo A-I by the isolated kidney were also studied. Radiolabeled HDL $(d=1.063-1.210)$ preparations obtained from subjects with high HDL cholesterol levels were incubated with VLDL for $24 \mathrm{~h}$ at $37^{\circ} \mathrm{C}$ to diminish apo $\mathrm{C}$ and apo $\mathrm{E}$ labeling. The HDL was reisolated by ultracentrifugation and incubated a second time for $24 \mathrm{~h}$ at $37^{\circ} \mathrm{C}$ with VLDL and $d>1.210$ fraction (as a source of cholesteryl ester transfer protein [CETP]). The triglyceride-enriched HDL was reisolated by ultracentrifugation and incubated a third time for $24 \mathrm{~h}$ at $37^{\circ} \mathrm{C}$ in the presence or absence of purified LPL (27) and HTGL (28). After one final reisolation by ultracentrifugation, the modified HDL was injected into the isolated kidney perfusion system as described above. Control HDL and triglyceride-enriched HDL, which were prepared identically to modified HDL except that they were not exposed to lipases, were studied in separate kidney perfusions.

We also assessed the effects of triglyceride enrichment and lipolysis on the association of apo A-I with HDL. HDL from subjects with high levels of plasma HDL cholesterol that had or had not been triglyceride enriched was incubated in the presence or absence of partially purified lipases as described above. LPL, HTGL, or the combination of the two lipases were used in these incubations. The unmodified or modified
HDL was then subjected to ultracentrifugation at $d=1.210$ for $24 \mathrm{~h}$. Radioactivity in the $d<1.210$ and $d>1.210$ fractions was examined to assess if any of the modifications affected the degree to which apo A-I remained bound to HDL particles.

Assays. Plasma and lipoprotein triglyceride and cholesterol levels were determined using enzymatic methods on an automated spectrophotometer (ABA-100; Abbott Laboratories, Chicago, IL). Our laboratory is standardized by the Centers for Disease Control and we participate in their ongoing quality control program. Apo A-I was measured by radioimmunoassay in plasma, HDL, and $d>1.210$ fractions (7). Our laboratory participated in the Centers for Disease Control apoprotein assay standardization study (29).

Statistics. Comparisons between whole- and exchange-labeled tracers were made using paired $t$ tests. Comparisons between groups with low and high HDL cholesterol levels were made using unpaired $t$ tests.

\section{Results}

Subjects. The clinical characteristics of the study patients are depicted in Table I. All subjects were males. The average age of the low HDL cholesterol group was $42 \mathrm{yr}$; the average age of three subjects with high HDL cholesterol levels was $35 \mathrm{yr}$. The group with low HDL cholesterol had a greater mean percent ideal body weight than the high HDL cholesterol group (112.8 \pm 16.3 vs. $94.1 \pm 12.7 \%$ ) Plasma lipids at the time of referral to our clinic or at the time of recruitment for the study are also presented in Table I. By design, all of the subjects in the low HDL cholesterol group had values below the 25 th percentile for their age whereas subjects in the high HDL cholesterol group had values above the 90th percentile for their age. Mean HDL cholesterol concentrations were $31 \pm 4.3$ and $72 \pm 4.5 \mathrm{mg} /$ $\mathrm{dl}$ in the two groups $(P<0.0001)$. Although total and LDL cholesterol values were similar in each group, triglyceride measurements were higher in the group with low HDL cholesterol ( $372 \pm 275$ vs. $92 \pm 24 \mathrm{mg} / \mathrm{dl} ; P<0.03$ using log values).

The mean lipid values for each patient during the study period are depicted in Table II. There was significant variation during the study period only in the measurements of plasma

Table I. Clinical Characteristics of Study Patients

\begin{tabular}{|c|c|c|c|c|c|c|}
\hline Subject & Age & $\%$ IBW* $^{*}$ & $\mathrm{TC}^{*}$ & $\mathrm{TG}^{*}$ & HDLC & LDLC \\
\hline & $y r$ & & & & & \\
\hline \multicolumn{7}{|l|}{ Low HDLC } \\
\hline 1 & 26 & 95.5 & 194 & 241 & 33 & 113 \\
\hline 2 & 42 & 133.2 & 355 & 269 & 30 & 271 \\
\hline 3 & 41 & 90.4 & 171 & 101 & 35 & 116 \\
\hline 4 & 57 & 118.0 & 215 & 353 & 31 & 109 \\
\hline 5 & 62 & 109.0 & 176 & 174 & 34 & 107 \\
\hline 6 & 28 & 112.3 & 238 & 897 & 22 & 58 \\
\hline 7 & 40 & 131.2 & 194 & 569 & 31 & 49 \\
\hline Mean \pm sd & $42 \pm 13$ & $112.8 \pm 16.3$ & $220 \pm 64$ & $372 \pm 275^{\ddagger}$ & $31 \pm 4.3^{8}$ & $118 \pm 73$ \\
\hline \multicolumn{7}{|l|}{ High HDLC } \\
\hline 8 & 45 & 108.5 & 217 & 115 & 72 & 122 \\
\hline 9 & 25 & 89.3 & 145 & 67 & 68 & 64 \\
\hline 10 & 35 & 84.5 & 192 & 95 & 77 & 96 \\
\hline Mean \pm sd & $35 \pm 10$ & $94.1 \pm 12.7$ & $185 \pm 37$ & $92 \pm 24$ & $72 \pm 4.5$ & $94 \pm 29$ \\
\hline
\end{tabular}

* \% IBW, Percent ideal body weight; TC, total cholesterol; TG, total triglycerides; HDLC, high density lipoprotein cholesterol; LDLC, low density lipoprotein cholesterol. ${ }^{\ddagger} P<0.03$, low vs. high HDLC. ${ }^{\S} P<0.0001$, low vs. high HDLC. 
Table II. Mean Lipid Values for Each Patient during the Study Period

\begin{tabular}{cclcc}
\hline Subject & \multicolumn{1}{c}{ TC* $^{*}$} & \multicolumn{1}{c}{ TG } & HDL & LDL \\
\hline Low HDLC & & & & \\
1 & $181 \pm 13$ & $355 \pm 253$ & $29 \pm 4$ & $83 \pm 25$ \\
2 & $343 \pm 49$ & $270 \pm 92$ & $28 \pm 3$ & $261 \pm 31$ \\
3 & $163 \pm 12$ & $123 \pm 22$ & $28 \pm 3$ & $82 \pm 3$ \\
4 & $229 \pm 13$ & $428 \pm 213$ & $32 \pm 3$ & $120 \pm 31$ \\
5 & $166 \pm 20$ & $266 \pm 37$ & $27 \pm 2$ & $86 \pm 20$ \\
6 & $199 \pm 10$ & $799 \pm 255$ & $19 \pm 5$ & $34 \pm 20$ \\
7 & $231 \pm 56$ & $790 \pm 266$ & $27 \pm 3$ & $43 \pm 17$ \\
High HDLC & & & & \\
8 & $210 \pm 8$ & $111 \pm 34$ & $70 \pm 5$ & $118 \pm 9$ \\
9 & $125 \pm 13$ & $97 \pm 22$ & $60 \pm 8$ & $47 \pm 10$ \\
10 & $210 \pm 10$ & $40 \pm 8$ & $86 \pm 8$ & $114 \pm 8$ \\
& & & & \\
\hline
\end{tabular}

* All lipids are mean \pm standard deviations for 18 measurements obtained during the 2-wk sampling period.

triglycerides in subjects with significant hypertriglyceridemia. The concentration of HDL triglyceride was similar in both groups of subjects (data not shown). Since the HDL cholesterol concentration was significantly lower in the subjects with low HDL levels, their HDL particles were relatively triglyceride enriched compared with the HDL present in the subjects with high HDL levels.

HDL turnover studies. The data were fitted adequately by a separate two-pool model for each tracer, with the constraint that the two tracers shared a single noncirculating pool. The mean residual errors of the fits ranged from 5.4 to $17.2 \%$ $($ mean $\pm \mathrm{SD}=10.5 \pm 3.7 \%)$ for the whole tracer and 4.2 to $11.4 \%(6.7 \pm 2.2 \%)$ for the exchange tracer. The adequacy of the six-parameter model was tested by fitting the data with an eight-parameter model allowing the noncirculating pools to be different for the two tracers. The fits of the data were not improved significantly, with the mean residual errors averaging $10.2 \pm 3.9 \%$ for the whole tracer and $6.2 \pm 2.4 \%$ for the exchange tracer. These data support the assumption of identical noncirculating pools and, in turn, validate the dual-tracer approach.

Because our hypothesis assumes that there are really two pools of circulating apo A-I, one that is exchangeable and one that is nonexchangeable, we also attempted to determine the proportion of total apo A-I that is present in each pool. To a first approximation, the partition of apo A-I would be described by the following:

whole tracer $\mathrm{FCR}=f \mathrm{FCR}_{\mathrm{E}}+(1-f) \mathrm{FCR}_{\mathrm{NE}}$,

where $f$ is the fraction of apo A-I that is exchangeable and the subscripts $\mathrm{E}$ and $\mathrm{NE}$ refer to exchangeable and nonexchangeable apo A-I, respectively. We know the whole tracer FCR, and the exchange tracer FCR is a measure of $\mathrm{FCR}_{\mathrm{E}}$. $\mathrm{FCR}_{\mathrm{NE}}$ cannot, however, be determined without knowledge of the exchangeable fraction, $f$. When we attempted to estimate $f$ by fitting the whole tracer data with a four-pool model (two pools of exchangeable apo A-I as determined by the exchange tracer, two pools of nonexchangeable apo A-I and identical noncirculating pools), we could not improve the fit nor could we estimate uniquely the value of $f$. We concluded that it was not possible to partition the whole tracer FCR and estimate nonexchange-

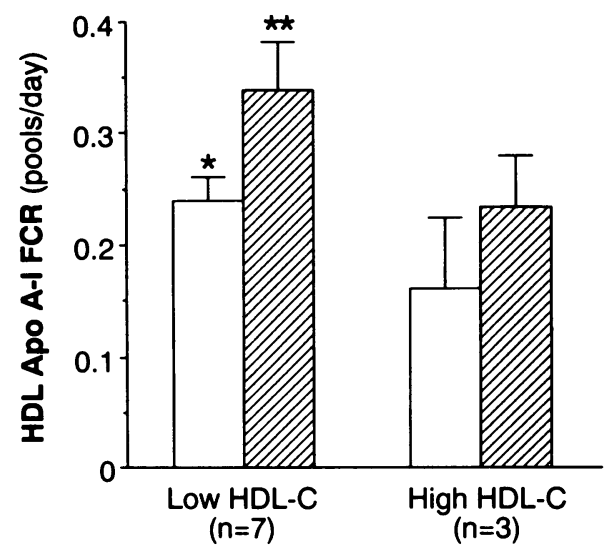

Figure 1. HDL apo A-I FCRs in subjects with low and high levels of HDL cholesterol. Open bars represent whole-labeled FCR and crosshatched bars represent exchange-labeled FCR. ${ }^{*} P<0.009$, low vs. high HDL-C group. ${ }^{* *} P<0.02$, low vs. high HDL-C group.

able apo A-I FCR uniquely. We can state that the nonexchangeable apo A-I FCR is smaller than the whole tracer FCR (since exchangeable apo A-I FCR is greater than whole tracer FCR), and that the greater the proportion of apo A-I that is exchangeable, the smaller the nonexchangeable apo A-I FCR.

Parameters describing HDL apo A-I metabolism are depicted in Fig. 1. In every subject, whether in the low or high HDL cholesterol group, the FCR of the exchange-labeled tracer was greater than the FCR of the whole-labeled tracer (Table III). The exchange-labeled FCR (mean \pm SD) was higher in the low HDL cholesterol group $\left(0.339 \pm 0.043 d^{-1}\right)$ than in the high HDL cholesterol group $\left(0.234 \pm 0.047 d^{-1} ; P\right.$ $<0.009$ ). Similarly, the whole-labeled FCR was greater in the low HDL cholesterol group $\left(0.239 \pm 0.023 d^{-1}\right)$ than in the high HDL cholesterol group $\left(0.161 \pm 0.064 d^{-1} ; P<0.02\right)$. These findings indicate that exchange labeling of HDL resulted in a tracer that described the kinetics of a more rapidly removed pool of apo A-I and that individuals with low levels of HDL cholesterol had a greater proportion of their total apo A-I in this pool.

Table III. Individual HDL Turnover Parameters for All Subjects

\begin{tabular}{ccccc}
\hline Subject & $\begin{array}{c}\text { Whole apo } \\
\text { A-I FCR }\end{array}$ & $\begin{array}{c}\text { Exchange apo } \\
\text { A-I* FCR }\end{array}$ & $\begin{array}{c}\text { HDL } \\
\text { apoA-I }\end{array}$ & $\begin{array}{c}d>1.210 \\
\text { apoA-I }\end{array}$ \\
\hline Low HDL & & & & \\
1 & 0.253 & 0.392 & $55.9 \pm 3.3$ & $14.7 \pm 1.6$ \\
2 & 0.200 & 0.268 & ND & ND \\
3 & 0.270 & 0.309 & ND & ND \\
4 & 0.226 & 0.357 & ND & ND \\
5 & 0.228 & 0.361 & $32.3 \pm 3.4$ & $6.6 \pm 1.6$ \\
6 & 0.247 & 0.372 & $55.3 \pm 3.7$ & $8.6 \pm 0.9$ \\
7 & 0.250 & 0.317 & $102.6 \pm 17.0$ & $13.6 \pm 1.5$ \\
High HDL & & & & \\
8 & 0.120 & 0.267 & $120.3 \pm 9.4$ & $6.8 \pm 0.9$ \\
9 & 0.235 & 0.255 & $100.8 \pm 14.0$ & $10.9 \pm 1.6$ \\
10 & 0.128 & 0.181 & $110.9 \pm 15.9$ & $5.6 \pm 1.2$
\end{tabular}

* FCR in $\mathrm{d}^{-1}$. $\quad{ }^{\ddagger}$ Apo A-I in $\mathrm{mg} / \mathrm{dl}$ : each value is the mean \pm SD of six to eight values obtained during the turnover study. 
The radioactivity recovered in the $d>1.210$ fraction in several of the turnover studies, calculated as a percent of total plasma radioactivity, is shown in Fig. 2. In every patient, whether from the low or high HDL cholesterol group, there was more radioactivity in the $d>1.210$ fraction derived from the exchange-labeled than from the whole-labeled tracer $(12.55$ \pm 4.95 vs. $1.02 \pm 0.38 \% ; P<0.003$ ). In addition, there was significantly more exchange-labeled $d>1.210$ radioactivity in the low HDL cholesterol $(16.5 \pm 3.6 \%)$ than in the high HDL cholesterol group $(8.6 \pm 1.0 \% ; P<0.02)$. There was also more than a twofold greater proportion of apo A-I, measured by radioimmunoassay in the $d>1.210$ fraction and reported as a percentage of HDL apo A-I concentration, in the low versus the high HDL cholesterol groups (Fig. 3 and Table III). These data provide further evidence that exchange-labeled apo A-I traces a more easily dissociated pool of apo A-I and that this pool makes up a greater proportion of total apo A-I in subjects with low levels of HDL cholesterol than in subjects with high HDL cholesterol concentrations.

To exclude a role for "isotope effects" in the outcomes of these in vivo experiments, in which ${ }^{131} I$ exchange-labeled tracers and ${ }^{125}$ I whole-labeled tracers were used in most of the studies, we carried out two turnover studies (subjects 2 and 4) with the tracers reversed. The HDL apo A-I FCRs of these two subjects with low HDL cholesterol levels fell well within the range of the rest of the group. In each case the exchange-labeled tracer was removed more rapidly than the whole-labeled tracer and the difference between the FCRs in subjects $2(\Delta$ $\left.=0.68 d^{-1}\right)$ and $4\left(\Delta=0.131 d^{-1}\right)$ were comparable to the mean difference between the FCRs of the two tracers for the entire group with low HDL cholesterol levels. In addition, because we used a single homologous apo A-I preparation that was labeled to a high specific activity for each of the exchange-labeled studies, we carried out one study with both homologous and autologous exchange-labeled tracers. The FCRs of two exchange-labeled tracers (using both homologous and autologous apo A-I with a ratio of specific activities $>10: 1$ ) that were injected into one subject with a low HDL cholesterol concentration were virtually identical; the autologous and homologous HDL apo A-I FCRs were both 0.26 pools per day.

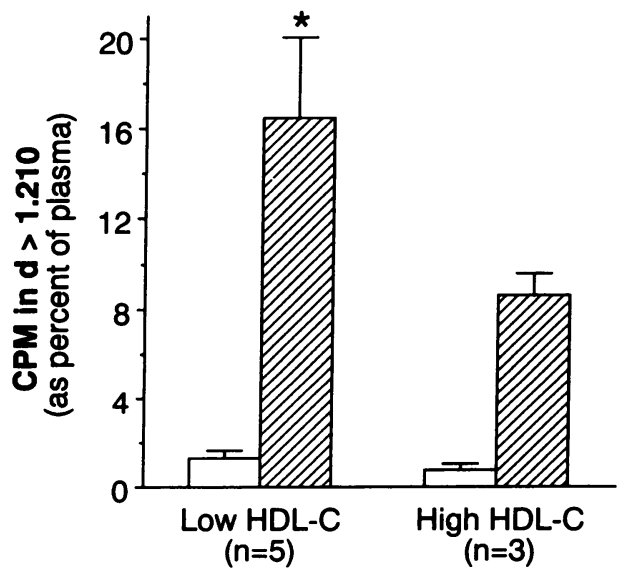

Figure 2. Radioactivity in $d>1.210$ fraction as a percent of plasma radioactivity in subjects with low and high levels of HDL cholesterol. Open bars represent whole-labeled tracer and cross-hatched bars represent exchange-radiolabeled tracer. ${ }^{*} P<0.02$, low vs. high HDL-C group.

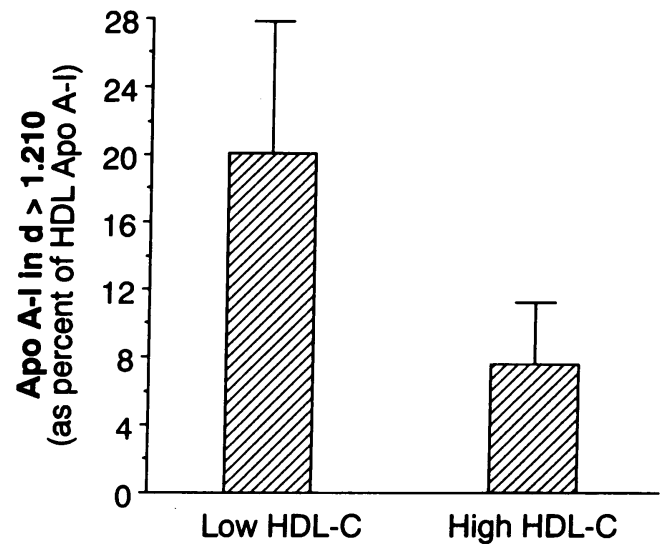

Figure 3. Apo A-I measured by radioimmunoassay in $d>1.210$ fraction as a percent of HDL apo A-I concentration in subjects with low and high levels of HDL cholesterol. Results are the means from four of seven subjects with low HDL cholesterol levels and all three subjects with high HDL cholesterol levels. Individual data are presented in Table III.

In vitro studies. The results of the study comparing the percent of exchange- and whole-labeled HDL tracers isolated in $d>1.210$ after incubation at $37^{\circ} \mathrm{C}$ for up to $2 \mathrm{wk}$ are depicted in Table IV. There were no reductions in the amount of radioactivity isolated in the top $1 \mathrm{ml}(d<1.210$ fraction $)$ over the 2-wk period of incubation. In addition, there were no significant differences between tracers. Although there was slightly more exchange-labeled ${ }^{131} \mathrm{I}$ tracer radioactivity in the $d>1.210$ fraction compared with the other three tracers, this difference was slight and it remained constant over the 2-wk period. These results indicate that the in vivo observations did not result from methodological artifacts related to labeling techniques or isotope effects.

Kidney perfusion studies. The data from the kidney perfusion studies, presented as cpm per $\mathrm{g}$ cortex, are depicted in Fig. 4. Twice as much apo A-I radioactivity accumulated in the rabbit kidney cortex during perfusion of HDL isolated from subjects with low HDL cholesterol levels $\left(1.07 \pm 0.31 \times 10^{4}\right.$ $\mathrm{cpm} / \mathrm{g}$ cortex ) compared with that accumulated during perfusion of HDL from individuals with high HDL cholesterol concentrations $\left(0.54 \pm 0.1 \times 10^{4} \mathrm{cpm} / \mathrm{g}\right.$ cortex; $\left.P<0.0025\right)$. After the kidneys were perfused and washed, the final amount of radioactivity in the effluent averaged $1.2 \%$ of that in the initial perfusate. In one experiment, a $>10$-fold greater amount of tracer was added to the perfusate and the percent of renal cortical uptake was similar to all other studies. Therefore, at the amounts of tracer used in the experiments depicted in Fig. 4, renal uptake was not saturated. Finally, uptake and accumulation of retinol binding protein was similar in all experiments.

Modification of $H D L$. Renal uptake of control, triglyceride-enriched, and hydrolyzed triglyceride-enriched HDL is shown in Fig. 5. When the triglyceride-enriched HDL, which had been exposed to both partially purified LPL and HTGL, was added to the kidney perfusate, three times the apo A-I accumulated in the cortex compared with the unmodified preparation $\left(1.7 \pm 0.8 \times 10^{4}\right.$ vs. $0.54 \pm 0.1 \times 10^{4} \mathrm{cpm} / \mathrm{g}$ cortex; $P$ $<0.007)$. Triglyceride enrichment of HDL alone did not affect uptake significantly $(0.74 \pm 0.3)$. These results paralleled those obtained when we perfused HDL from individuals with low and high HDL cholesterol levels and suggest that triglyceride- 
Table IV. Results of 2-wk Incubation Study Comparing Exchange- and Whole-labeled HDL Tracers with either ${ }^{131} I$ or ${ }^{125} I$

\begin{tabular}{|c|c|c|c|c|c|}
\hline \multirow{2}{*}{$\frac{\text { Day }}{0}$} & \multirow{2}{*}{$\frac{\text { Aliquot }^{*}}{1}$} & \multicolumn{2}{|c|}{$W h-{ }^{131} I / E x-125 I^{\ddagger}$} & \multicolumn{2}{|c|}{$W h-{ }^{-125} I / E x-{ }^{131} I$} \\
\hline & & 91 & 92 & 92 & 83 \\
\hline & 2 & 6 & 5 & 6 & 9 \\
\hline & 3 & 3 & 3 & 2 & 8 \\
\hline \multirow[t]{3}{*}{3} & 1 & 87 & 88 & 92 & 88 \\
\hline & 2 & 10 & 9 & 6 & 9 \\
\hline & 3 & 3 & 3 & 2 & 3 \\
\hline \multirow[t]{3}{*}{6} & 1 & 91 & 92 & 94 & 88 \\
\hline & 2 & 7 & 6 & 4 & 7 \\
\hline & 3 & 2 & 2 & 2 & 5 \\
\hline \multirow[t]{3}{*}{8} & 1 & 90 & 91 & 79 & 72 \\
\hline & 2 & 8 & 7 & 19 & 22 \\
\hline & 3 & 2 & 2 & 2 & 6 \\
\hline \multirow[t]{3}{*}{10} & 1 & 90 & 89 & 92 & 84 \\
\hline & 2 & 8 & 9 & 6 & 10 \\
\hline & 3 & 2 & 2 & 2 & 6 \\
\hline \multirow[t]{3}{*}{14} & 1 & 90 & 90 & 94 & 87 \\
\hline & 2 & 8 & 8 & 5 & 8 \\
\hline & 3 & 2 & 2 & 1 & 5 \\
\hline \multirow[t]{3}{*}{18} & 1 & 88 & 90 & 91 & 82 \\
\hline & 2 & 9 & 7 & 7 & 10 \\
\hline & 3 & 3 & 3 & 2 & 8 \\
\hline \multirow[t]{3}{*}{20} & 1 & 89 & 91 & 92 & 82 \\
\hline & 2 & 8 & 6 & 6 & 10 \\
\hline & 3 & 3 & 3 & 2 & 8 \\
\hline
\end{tabular}

HDL was both whole and exchange labeled with both ${ }^{125} \mathrm{I}$ and ${ }^{131} \mathrm{I}$, and two separate incubations were carried out at $37^{\circ} \mathrm{C}$ for $2 \mathrm{wk}$, using 131-I whole-labeled tracer and 125-I exchange-labeled tracer in one incubation, and 125-I whole-labeled tracer and 131-I exchangelabeled tracer in the other. Every $2 \mathrm{~d}$, an aliquot was taken from each incubation and subjected to ultracentrifugation at $d=1.210$ for 24

h. * Samples were spun in a 50.3 Ti ultracentrifuge tube with a total volume of $6 \mathrm{ml}$ in each tube. Number represents the aliquot taken from the tube (i.e., $1=$ top $1 \mathrm{ml}, 2=$ next $2 \mathrm{ml}, 3=$ bottom $3 \mathrm{ml}$ ). We have defined the top $1 \mathrm{ml}$ as $d<1.210$ and the bottom $3 \mathrm{ml}$ as $d$ $>1.210$. ${ }^{\ddagger} \mathrm{Wh}$, whole-labeled; Ex, exchange-labeled. Number represents the cpm in that aliquot as a percent of the total counts in the tube.

enrichment of HDL followed by lipolysis could be important in vivo.

Modified and unmodified HDL were also subjected to ultracentrifugation at $d=1.210$ to determine whether the degree of dissociation of apo A-I was affected by triglyceride enrichment and lipolysis. The results are presented in Table V. Exposing unmodified HDL isolated from subjects with high HDL cholesterol levels to lipases did not alter the amount of radioactivity found in the $d<1.210$ and $d>1.210$ fractions. Similarly, triglyceride enrichment alone did not affect the distribution of radioactivity. However, when triglyceride-enriched HDL was exposed to LPL, significantly more radioactivity was found in the $d>1.210$ fraction. This effect was increased by using a combination of LPL and HTGL. Therefore, triglyceride enrichment of HDL followed by incubation with LPL or the combination of LPL and HTGL resulted in the greatest dissociation of apo A-I in the ultracentrifuge.

The different modified HDL preparations used in the ex-

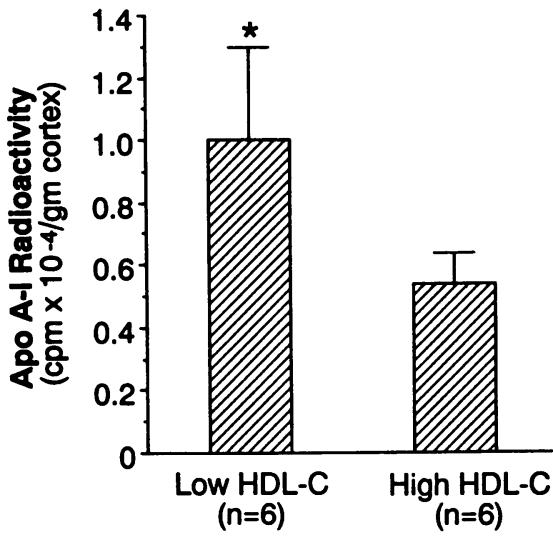

Figure 4. Results of kidney perfusion studies in which kidneys isolated from rabbits were perfused with whole-labeled ${ }^{125} \mathrm{I}$-HDL from subjects with low or high levels of HDL cholesterol. Accumulation of apo A-I in the kidney was quantified by determining the radioactivity in the cortex, expressed per $\mathrm{g}$ tissue, normalized to initial radioactivity in the perfusate. ${ }^{*} P<0.0025$, low vs. high HDL cholesterol group.

periment described above were examined for size differences by gel filtration on a Sephadex G-150 column. Fig. 6 compares the gel-filtration pattern of triglyceride-enriched HDL that had been exposed to both lipases with control HDL. The modified HDL was significantly smaller than the unmodified preparation. No clear peak eluting from the Sephadex G-150 column at a size consistent with free apo A-I was noted, however. Similar reductions in HDL size after triglyceride enrichment and lipase-induced hydrolysis were demonstrated on nondenaturing gels (data not shown).

\section{Discussion}

Individuals with low plasma levels of HDL cholesterol almost uniformly have concomitant reductions in plasma levels of apo A-I, the major apolipoprotein in HDL. Although the majority of individuals with low levels of apo A-I have increased frac-

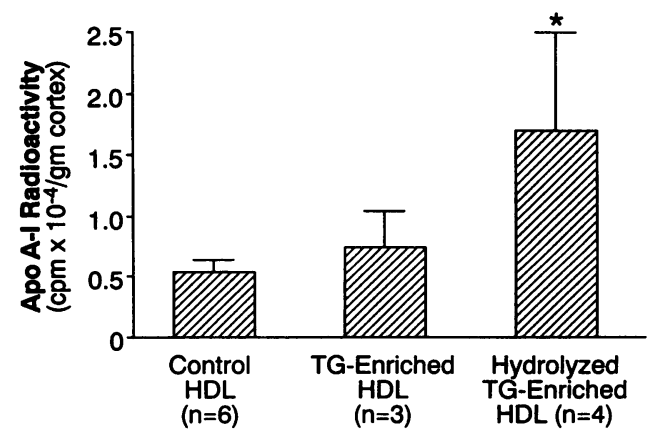

Figure 5. Results of kidney perfusion studies using unmodified or modified HDL. HDL from subjects with high levels of plasma HDL cholesterol that had or had not been triglyceride enriched was incubated in the presence or absence of partially purified LPL and HTGL. The HDL preparations were then perfused through kidneys isolated from rabbits. Accumulation of apo A-I in the kidney was quantified by determining the radioactivity in the cortex, expressed per $\mathrm{g}$ tissue, normalized to initial radioactivity in the perfusate. ${ }^{*} P<0.007$, hydrolyzed TG-enriched HDL vs. control HDL. 
Table $V$. Results of Ultracentrifugation of Modified and Unmodified HDL That Was or Was Not Subjected to Lipase

\begin{tabular}{llll}
\hline \multicolumn{1}{c}{ Sample* } & $1^{\ddagger}$ & 2 & 3 \\
\hline CHDL & 81 & 14 & 5 \\
CHDL + HL & 83 & 12 & 5 \\
CHDL + LPL & 82 & 13 & 5 \\
CHDL + HL + LPL & 81 & 14 & 5 \\
TGHDL & 81 & 13 & 6 \\
TGHDL + HL & 80 & 14 & 6 \\
TGHDL + LPL & 65 & 20 & 15 \\
TGHDL + HL + LPL & 58 & 23 & 19 \\
& & &
\end{tabular}

HDL from subjects with high levels of plasma HDL cholesterol that had or had not been triglyceride enriched was incubated in the presence or absence of partially purified lipases. LPL, HTGL, or the combination of the two lipases were used in these incubations. The unmodified or modified HDL was then subjected to ultracentrifugation at $d=1.210$ for $24 \mathrm{~h}$. Radioactivity in the $d<1.210$ and $d$ $>1.210$ fractions was examined to assess if any of the modifications affected the degree to which apo A-I remained bound to HDL particles. * CHDL, control HDL; HL, hepatic lipase; LPL, lipoprotein lipase; TGHDL, triglyceride-enriched HDL. ${ }^{\ddagger}$ Samples were spun in a $50.3 \mathrm{Ti}$ ultracentrifuge tube with a total volume of $6 \mathrm{ml}$ in each tube. Number represents the aliquot taken from the tube (i.e., 1 $=$ top $1 \mathrm{ml}, 2=$ next $2 \mathrm{ml}, 3=$ bottom $3 \mathrm{ml}$ ). We have defined the top $1 \mathrm{ml}$ as $d<1.210$ and the bottom $3 \mathrm{ml}$ as $d>1.210$.

tional clearance of apo A-I from plasma $(5-7,30)$, the exact mechanisms underlying accelerated HDL apo A-I catabolism have not been delineated. On the basis of the demonstrated ability of apo A-I to exchange between lipoprotein particles (10) and the finding that apo A-I can exist "free" in plasma (11-14), we proposed that apo A-I could bind to HDL with a

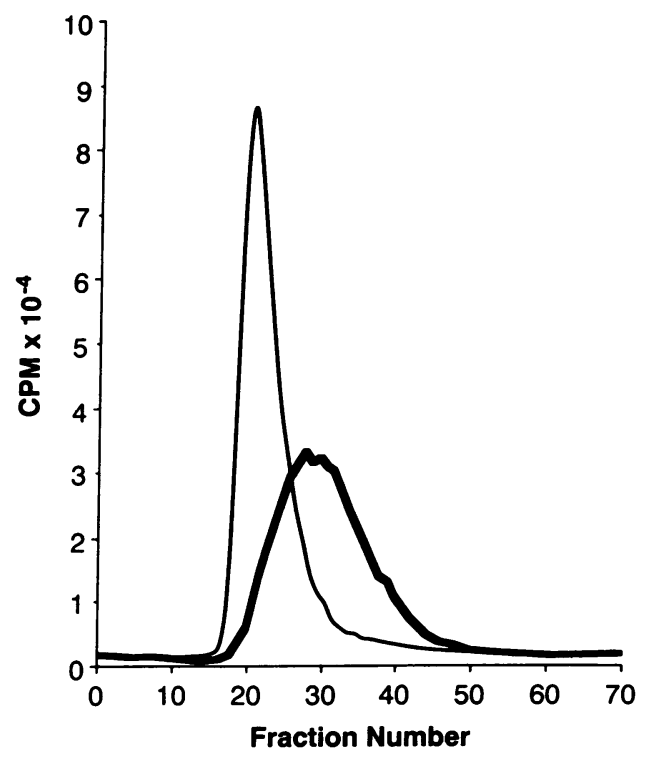

Figure 6. Results of Sephadex G-150 column elution. Regular line represents unmodified HDL; heavy line represents triglycerideenriched HDL exposed to both LPL and HTGL. Results indicate that TG enrichment followed by lipolysis results in a reduction in size of HDL. spectrum of affinities and that individuals with low plasma HDL cholesterol levels would have a greater proportion of their apo A-I in an easily dissociable state.

To test this hypothesis, we chose a dual-tracer approach to study HDL apo A-I metabolism in subjects with low and high levels of plasma HDL cholesterol. Whole labeling of HDL was used to label all of the apo A-I molecules on each HDL particle; exchange labeling of HDL was used in an attempt to preferentially label the least tightly bound, most easily dissociable apo A-I molecules. In this manner we hoped to simultaneously trace the average kinetics of all HDL apo A-I (whole-labeled tracer) and the specific kinetics of loosely bound HDL apo A-I (the exchange-labeled tracer). It was not clear initially if we could achieve this goal, however, because the tracers might, over the course of the 2-wk study, become more like each other. Thus, as HDL particles were constantly remodeled in vivo, some exchange-labeled apo A-I molecules might become less dissociable and the exchange-labeled tracer would come to resemble the whole-labeled tracer. Although some remodeling of the exchange-labeled tracer most certainly occurred, our demonstration that the exchange-labeled FCR was significantly higher than whole-labeled FCR in every subject studied, whether they had high or low plasma HDL cholesterol concentrations, indicated that remodeling was not fast enough to overcome the initial differences in the two tracers. Similarly, in all subjects, ultracentrifugation of plasma at $d=1.210$ was associated with greater dissociation of exchange-labeled apo A-I from HDL particles than of whole-labeled apo A-I. These results supported the view that we would be able to distinguish between pools of apo A-I with different physical and biological characteristics by our dual-labeling approach.

The test of our hypothesis was, therefore, if the different tracers would distinguish between our two subject groups. Our observation that the exchange-labeled FCRs were significantly higher in the low versus the high HDL cholesterol group indicated that a greater proportion of exchange-labeled apo A-I was in an exchangeable state throughout the sampling period in the former group of subjects. The greater whole-labeled FCR in the low versus the high HDL cholesterol group provided further support for the view that more apo A-I molecules were in an exchangeable, more easily dissociable state in the group with low HDL cholesterol concentrations.

Additional support for our hypothesis derived from the observation that although the proportion of apo A-I dissociating from HDL during ultracentrifugation of exchange-labeled tracer was much greater than that of whole-labeled tracer in all subjects, this difference was more pronounced in the low versus the high HDL cholesterol group. Thus, the two HDL tracers demonstrated differences between the two subject groups that were compatible with the hypothesis that individuals with low levels of HDL cholesterol have a greater proportion of their apo A-I in an easily dissociable form. This conclusion is supported further by the finding of a greater proportion of HDL apo A-I measured by radioimmunoassay in the $d>1.210$ fraction in the low versus the high HDL cholesterol groups.

Several groups of investigators have compared the catabolism of whole- and exchange-labeled HDL, and published results are conflicting. Shepherd et al. (31), studying three subjects, also found higher FCRs with exchange-labeled compared with whole-labeled HDL when they used radiolabeling protocols similar to those we used in the present studies. In contrast, Schaefer et al. (32) found no differences between exchange- 
and whole-labeled tracer FCRs in two subjects when the exchange-labeled particles were generated in vivo by injection of radiolabeled apo A-I directly into plasma as radiolabeled autologous apo A-I. More recently, Vega et al. (33) studied 11 individuals with a range of HDL cholesterol levels and found no difference in apo A-I FCRs after injecting whole-labeled HDL and radiolabeled autologous apo A-I into subjects. Vega et al. labeled $1 \mathrm{mg}$ of apo A-I, which is much more mass than is usually labeled in such studies (we routinely labeled $20 \mu \mathrm{g}$ of apo A-I ), and suggested that reduced radiation damage of their exchange-labeled tracer accounted for the differences between their results and those of Shepherd et al. (31) (and, by inference, our results). These authors also argued that differences between homologous and autologous apo A-I molecules might result in differences in the FCRs of whole- and exchange-labeled tracers. In one of our patients, we used both homologous and autologous apo A-I to create two separate exchange-labeled tracers. We radiolabeled $\sim 20 \mu \mathrm{g}$ of homologous apo A-I and $\sim 500 \mu \mathrm{g}$ of autologous apo A-I. The FCRs of the two exchange-labeled tracers were identical in this subject. We believe, therefore, that neither the source of apo A-I nor radiation damage played a role in the differences we observed in the rest of our subjects between the apo A-I FCRs determined with whole- and exchange-labeled tracers.

A potential reason for the difference between our results and those of Vega et al. (33) may derive from the methods used to isolate HDL. Vega et al. used HDL of $d=1.090-1.210$ for direct, whole-radiolabeling, whereas we used the whole spectrum of HDL from $d=1.063$ to $d=1.210$. We have observed by nondenaturing gels that exchange labeling preferentially labels smaller HDL (data not shown) whereas direct, whole labeling appears to label all HDL size populations. It is possible, therefore, that Vega et al., by isolating HDL at $d=1.090$ 1.210, whole labeled a population of small HDL particles that was similar to the population preferentially labeled when they injected pure apo A-I tracer intravenously. This conclusion is supported by the presence of a high percentage of radioactivity in the $d>1.210$ fraction derived from the whole-labeled tracer in their studies; that percent was similar to what we observed in the present studies with our exchange-labeled tracer and to what was reported by Brinton et al. (30) who injected radiolabeled apo A-I directly in their investigations. In contrast, we observed much less radioactivity in $d>1.210$ from our wholelabeled HDL tracers.

Could the differences between the two tracers in vivo be due to an isotope effect? We began our studies using ${ }^{131} \mathrm{I}$ for exchange labeling because the much longer half-life of ${ }^{125} \mathrm{I}$ was better suited to the time-consuming isolation of apo A-I from the whole-labeled tracer by FPLC. Khouw et al. (25) recently described studies in which ${ }^{131}$ I-LDL injected into guinea pigs had greater FCRs than ${ }^{125} \mathrm{I}-\mathrm{LDL}$, and the difference between isotopes increased if more time was allowed to pass between labeling and injection of tracer. Hence, radiation damage to HDL labeled with ${ }^{131}$ I could have been the basis for higher FCRs and increased radioactivity in $d>1.210$ observed with the exchange-labeled tracers in our studies. We tested for this effect by reversing the isotopes in two subjects. The results indicated that there was no significant isotope effect in our studies. In addition, we always reisolated the exchange-labeled tracer by ultracentrifugation and injected it within $24 \mathrm{~h}$ of labeling to insure against artifacts associated with damaged particles. Finally, our in vitro incubation studies, in which we used each isotope to both whole and exchange label HDL, did not demonstrate any significant differences between the association of differentially radiolabeled apo A-I to HDL particles.

If accelerated apo A-I catabolism in individuals with low HDL cholesterol levels occurs because a greater proportion of their apo A-I molecules is more loosely associated with HDL particles, why is this so? Furthermore, if "free" apo A-I is rapidly cleared from plasma, is it taken up by any particular organ or tissue? To address these questions, we carried out a series of studies using an isolated kidney perfusion system. HDL apo A-I from subjects with low HDL cholesterol levels was taken up to a greater extent by the kidney than HDL apo A-I from high HDL cholesterol subjects. In addition, when HDL isolated from individuals with high plasma levels of HDL cholesterol was triglyceride enriched and subjected to hydrolysis by LPL and HTGL, more apo A-I was removed by the kidney. The results of the kidney perfusion studies with modified HDL were consistent with our observation that more radioactivity was found in the $d>1.210$ fraction when triglyceride-enriched hydrolyzed HDL (originally isolated from subjects with high HDL cholesterol levels) was subjected to ultracentrifugation. Together, these results suggest that individuals with low plasma levels of HDL cholesterol, who also have small, relatively triglyceride-enriched HDL particles, have more "free" apo A-I available to be cleared by the kidney.

The role of the kidney in apo A-I metabolism was first noted by Glass et al. (8) who directly labeled whole HDL with the "trapped ligand" ${ }^{125}$ I-tyramine-cellobiose and showed that accumulation of the tracer was proportional to the labeling of apo A-I on the particles. In that study (8) the authors also demonstrated the presence of immunoreactive apo A-I in tubular epithelial cells. In a later report, Glass et al. (9) reported that the rat kidney could accumulate apo A-I without necessarily taking up the entire HDL particle. Saku et al. (16) demonstrated that isolated kidneys could filter and reabsorb apo A-I. All of these results support the view that free apo A-I, rather than whole HDL, is cleared by the glomerulus and degraded by tubular cells. More recently, we demonstrated that monkeys made acutely hypertriglyceridemic by inhibition of LPL developed marked reductions in HDL cholesterol and apo A-I levels and that increased apo A-I radioactivity was accumulated in the kidneys of those animals after injection of whole-radiolabeled HDL (19). Neary and Gowland reported increased plasma free apo A-I in individuals with either renal disease (15) or hypertriglyceridemia (11).

Our studies suggest that increased renal clearance of apo A-I occurs when HDL that is cholesterol depleted and relatively enriched in triglycerides interacts with plasma LPL and/ or HTGL. We believe that the reduction in HDL size resulting from such interactions leads to increased apo A-I dissociation from HDL. Although, under our in vitro conditions, we were able to demonstrate a reduction in HDL size after hydrolysis of triglyceride-enriched particles with lipases, we did not find free apo A-I by gel filtration. Clay and colleagues (34) have demonstrated the generation of free apo A-I from HDL, using slightly different incubation conditions. It is interesting that in our studies we achieved significant effects only when both LPL and HTGL were used in the incubations. Less effect was attained with LPL alone, and virtually no effect was observed when we used HTGL alone. In contrast, Clay et al. (34) observed the greatest effect with HTGL alone whereas Newnham et al. (35) found that LPL reduced the effect of HTGL. The disparities in 
these results are not easily resolved, but Clay et al. (34) and Newnham et al. (35) incubated lipases with serum, which included VLDL as a source of triglyceride, rather than with isolated, triglyceride-enriched HDL. Therefore, the presence of VLDL and/or other factors in serum might be required for the complete dissociation of apo A-I from HDL. Nonetheless, the observation that triglyceride-enriched HDL that has been exposed to lipase is smaller than unmodified HDL has been consistent.

In conclusion, we believe that the present studies show that individuals with low plasma levels of HDL cholesterol have increased apo A-I FCRs because they have an increased pro-

\section{A Low Levels of VLDL TG}

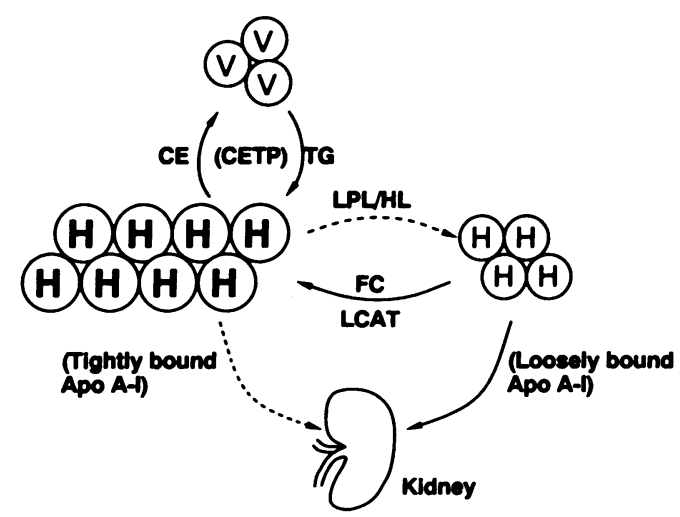

B High Levels of VLDL TG

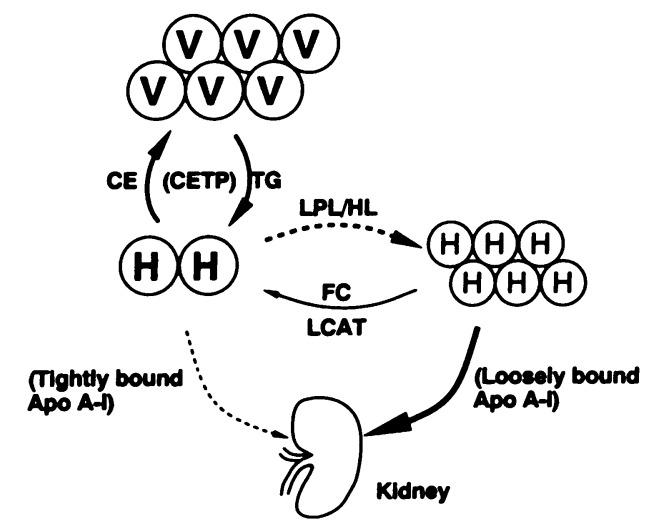

Figure 7. Proposed relationship between plasma levels of triglyceriderich lipoproteins, HDL cholesterol levels, and HDL apo A-I catabolism. $(A)$ In individuals with low plasma levels of triglyceride-rich lipoproteins there is reduced CETP-mediated exchange of HDL cholesteryl ester for VLDL triglycerides. This results in a distribution of HDL particles that favors cholesteryl ester-enriched HDL. Since apo A-I is more tightly bound to cholesteryl ester-enriched particles there will be less apo A-I in an easily dissociable pool that can be catabolized by the kidney. Total apo A-I FCR will be low, apo A-I levels will be increased, and there will be more HDL particles in the circulation. $(B)$ In individuals with high plasma levels of triglyceride-rich lipoproteins there is increased CETP-mediated exchange of core lipids. This results in a distribution of HDL particles that favors cholesteryl ester-depleted HDL. Since apo A-I is less tightly bound to cholesteryl ester-depleted particles, there will be more apo A-I in an easily dissociable pool that can be catabolized by the kidney. Total apo A-I FCR will be high, apo A-I levels will be decreased, and there will be fewer HDL particles in the circulation. portion of their HDL apo A-I in a more easily dissociable pool that can be more rapidly cleared by the kidney. In Fig. 7, a schema of HDL apo A-I metabolism is proposed to explain differences between individuals with high (Fig. $7 A$ ) or low (Fig. $7 B$ ) plasma concentrations of HDL cholesterol. Individuals with low plasma levels of HDL cholesterol have increased CETP-mediated exchange of HDL cholesterol ester for VLDL triglyceride, which results in a triglyceride-enriched HDL that is an excellent substrate for HL and/or LPL. After hydrolysis of core triglyceride, apo A-I binds less well to the surface of the particle. Whether this lipid-depleted HDL can be characterized by its size or buoyant density $\left(\mathrm{HDL}_{2}\right.$ vs. $\left.\mathrm{HDL}_{3}\right)$, by its specific apolipoprotein composition (apo A-I only vs. apo A-I:apo AII), or by its electrophoretic mobility (alpha vs. prebeta) remains to be determined. In any event, our data indicate that this loosely bound apo A-I more readily dissociates from HDL and is available for rapid clearance from plasma via glomerular filtration. Support for this conclusion can be found in reports by both Neary and Gowland ( 11 ) and Schonfeld et al. (12) of increased free apo A-I in plasma from individuals with hypertriglyceridemia. Future research will be aimed at determining if a particular HDL subclass is characterized by loosely bound, easily dissociable apo A-I and at more clearly discerning the physical and/or chemical alterations that allow a greater proportion of apo A-I to exist in that state.

\section{Acknowledgments}

We thank Ms. Colleen Ngai, Ms. Xiao-Jie Wang, and Mrs. Minnie Myers for their excellent technical assistance; the nurses of the Irving General Clinical Research Center for assistance with blood drawing; and Mrs. Wahida Karmally and her staff for their help with dietary and nutritional counseling.

This work was supported by the following grants: NHLBI HL 21006, HL 36000, HL 07343; DRR RR 645; a grant from the Council for Tobacco Research, USA and a grant-in-aid from the American Heart Association-Bristol Myers Squibb. Dr. Goldberg is a recipient of an Established Fellowship from the American Heart Association, New York City Affiliate.

\section{References}

1. Miller, N. E., D. S. Thelle, O. H. Forde, and O. D. Mjos. 1977. The Tromso heart study. High density lipoprotein and coronary heart disease: a prospective case control study. Lancet. 8019:965-968.

2. Castelli, W. P., R. J. Garrison, P. W. Wilson, R. D. Abbott, S. Kalousdian, and W. B. Kannel. 1986. Incidence of coronary heart disease and lipoprotein cholesterol levels: the Framingham study. JAMA (J. Am. Med. Assoc.). 256:2835-2838.

3. Gordon, D. J., J. Knoke, J. L. Probstfield, R. Superko, and H. A. Tyroler. 1986. High density lipoprotein cholesterol and coronary heart disease in hypercholesterolemic men: the lipid research clinics coronary primary prevention trial. Circulation. 74:1217-1225.

4. Brunzell, J. D., A. D. Sniderman, J. J. Albers, and P. O. Kwiterovich, Jr. 1984. Apoproteins B and A-1 and coronary artery diseases in humans. Arteriosclerosis. 4:79-83.

5. Nicoll, A., N. E. Miller, and B. Lewis. 1980. High density lipoprotein metabolism. Adv. Lipid Res. 17:54-106.

6. Brinton, E. A., S. Eisenberg, and J. L. Breslow. 1991. Increased apo A-I and apo A-II fractional catabolic rate in patients with low high density lipoproteincholesterol levels with or without hypertriglyceridemia. J. Clin. Invest. 87:536544.

7. Le, N.-A., and H. N. Ginsberg. 1988. Heterogeneity of apolipoprotein A-I metabolism in subjects with reduced plasma concentrations of high density lipoprotein cholesterol. Metab. Clin. Exp. 37:614-617.

8. Glass, C., R. C. Pittman, Keller, G. A., and D. Steinberg. 1983. Tissue sites of degradation of apoprotein A-I in the rat. J. Biol. Chem. 258:7161-7167.

9. Glass, C., R. C. Pittman, M. Civen, and D. Steinberg. 1983. Uptake of high 
density lipoprotein-associated apoprotein A-I and cholesterol esters by 16 tissues of the rat in vivo and by adrenal cells and hepatocytes in vitro. J. Biol. Chem 260:744-750.

10. Schaefer, E. J., D. M. Foster, L. L. Jenkins, R. T. Lindgren, M. Berman, R. I. Levy, and H. B. Brewer, Jr. 1979. The composition and metabolism of high density lipoprotein subfractions. Lipids. 14:511-522.

11. Neary, R. H., and E. Gowland. 1987. Stability of free apolipoprotein A-1 concentration in serum, and its measurement in normal and hyperlipidemic subjects. Clin. Chem. 33:1163-1169.

12. Schonfeld, G., A. Bailey, and R. Steelman. 1978. Plasma, apolipoprotein A-I and A-II levels in hyperlipidemia. Lipids. 13:951-959.

13. Gebhardt, D. O. I. M. Schicht, and L. C. Paul. 1984. The immunochemical determination of apolipoprotein A, total apoplipoprotein A-I and 'free' apolipoprotein A-I in serum of patients on chronic haemodialysis. Ann. Clin. Bio chem. 21:301-305.

14. Daerr, W. H., U. Minzlaff, and H. Greten. 1986. Quantitative determination of apolipoprotein A-I in high-density lipoproteins and 'free' apolipoprotein A-I by two-dimensional agarose gel lipoprotein: 'rocket' immunoelectrophoresis of human serum. Biochim. Biophys. Acta. 879:134-139.

15. Neary, R. H., and E. Gowland. 1988. The effect of renal failure and haemodialysis on the concentration of free apolipoprotein A-1 in serum and the implications for the catabolism of high-density lipoproteins. Clin. Chim. Acta. 171:239-245.

16. Saku, K., G. S. Reddy, B. A. Hynd, and M. L. Kashyap. 1984. Renal handling of high-density lipoproteins by isolated perfused kidneys. Metab. Clin. Exp. 33:432-438.

17. Gomo, Z. A., and L. O. Henderson. 1988. High-density lipoprotein apolipoproteins in urine. II. Enzyme-linked immunoassay of apolipoprotein A-I. Clin. Chem. 34:1781-1786.

18. deMendoza, S. G., M. L. Kashyap, C. Y. Chen, and R. F. Lutmer. 1976 High density lipoproteinuria in nephrotic syndrome. Metab. Clin. Exp. 25:11431149.

19. Goldberg, I. J., W. S. Blaner, T. M. Vanni, M. Moukides, and R. Ramakrishnan. 1990. Role of lipoprotein lipase in the regulation of high density lipoprotein apolipoprotein metabolism. Studies in normal and lipoprotein lipaseinhibited monkeys. J. Clin. Invest. 86:463-473.

20. McFarlane, A. S. 1958. Efficient trace labeling of proteins with iodine Nature (Lond.). 182:153.

21. Sheperd, J., A. M. Gotto, Jr., O. D. Taunton, M. J. Caslake, and E. Farish. 1977. The in vitro interaction of human apolipoprotein A-I and high density lipoproteins. Biochim. Biophys. Acta. 489:486-501.
22. Havel, R. A., H. A. Eder, and J. H. Bragdon. 1955. The distribution and chemical composition of ultracentrifugally separated lipoproteins in human serum. J. Clin. Invest. 34:1345-1353.

23. Lowry, O. H., N. J. Rosebrough, A. L. Farr, and R. J. Randall. 1951. Protein measurement with the Folin phenol reagent. J. Biol. Chem. 193:265275.

24. Polacek, D., C. Edelstein, and A. M. Scanu. 1981. Rapid fractionation of human high density apolipoproteins by high performance liquid chromatography. Lipids. 16:927-929.

25. Khouw, A. S., S. Parthasarathy, and J. L. Witztum. 1991. Exposure of low density lipoprotein to radiation initiates lipid peroxidation and alters metabolic behavior. Clin. Res. 39:62A. (Abstr.)

26. Maack, T. 1980. Physiological evaluation of the isolated perfused rat kidney. Am. J. Physiol. 238:F71-F78.

27. Socorro, L., C. C. Green, and R. L. Jackson. 1985. Proparation of a homogeneous and stable form of bovine milk lipoprotein lipase. Prep. Biochem. 15:133-143.

28. Ehnholm, C., H. Greten, and W. V. Brown. 1974. A comparative study of post-heparin lipolytic activity and a purified hyman plasma triacylglycerol lipase. Biochim. Biophys. Acta. 360:68-77.

29. Smith, S. J., G. R. Cooper, L. O. Henderson, et al. 1988. An international collaborative study on standardization of apolipoproteins A-I and B. Clin. Chem. 33:2240-2249.

30. Brinton, E. A., S. Eisenberg, and J. L. Breslow. 1988. Elevated high density lipoprotein cholesterol levels correlate with decreased apolipoprotein A-I and A-II fractional catabolic rate in women. J. Clin. Invest. 84:262-269.

31. Shepherd, J., C. J. Packard, A. M. Gotto, and D. O. Taunton. 1978. A comparison of two methods to investigate the metabolism of human apolipoproteins A-I and A-II. J. Lipid Res. 19:656-661.

32. Schaefer, E. J., L. A. Zech, and L. L. Jenkins. 1982. Human apolipoprotein A-I and A-II metabolism. J. Lipid Res. 23:850-862.

33. Vega, G. L., H. Gylling, A. V. Nichols, and S. M. Grundy. 1991. Evaluation of a method for study of kinetics of autologous apolipoprotein A-I. J. Lipid Res. 32:867-875.

34. Clay, M. A., H. H. Newnham, and P. J. Barter. 1991. Hepatic lipase promotes a loss of apolipoprotein A-I from triglyceride-enriched human high density lipoproteins during incubation in vitro. Arteriosclerosis. 11:415-422.

35. Newnham, H. H., G. J. Hopkins, S. Devlin, and P. J. Barter. 1990. Lipoprotein lipase prevents the hepatic lipase-induced reduction in particle size of high density lipoproteins during incubation of human plasma. Atherosclerosis. 82:167-176. 